\title{
Preparation and Purification of Porcine Circovirus Type 2 Chimeric Virus-Like Particles by a New Affinity Purification System
}

\author{
Jie Zhang ${ }^{1}$, Tushuai $\mathrm{Li}^{2}$, Zhihong Liu ${ }^{2}$, Hongtao $\mathrm{Li}^{3}$, Yiqi $\mathrm{Gu}^{4}$, Cheng Zhu ${ }^{5}$, Ying $\mathrm{Su}^{5}$, \\ Xiang $\mathrm{Hu}^{5}$, Jingyuan $\mathrm{Xu}^{5}$, Wen Dong ${ }^{5}$, and Haibin Zhang ${ }^{6}$ \\ ${ }^{1}$ Affiliation not available \\ ${ }^{2}$ Nanjing University \\ ${ }^{3}$ Animal Husbandry and Fisheries Division, 857 farm \\ ${ }^{4}$ Animal Health Supervision Institute in Wuzhong District \\ ${ }^{5}$ Changshu Institute of Technology \\ ${ }^{6}$ Nanjing Agricultural University
}

May 7, 2020

\begin{abstract}
Porcine epidemic diarrhea virus (PEDV) causes severe watery diarrhea with dehydration and high mortality in pigs, bringing huge economic losses to the swine industry worldwide. The current prevention of PEDV is mainly concentrated on inactivated vaccines. In this study, two novel B cell epitopes S1D5 (residues 744-759) and S1D6 (residues 756-771) of PEDV were displayed on capsid (Cap) protein of porcine circovirus type 2 (PCV2) in Escherichia coli (E. coli) expression system. The recombinant protein Cap-S1D can self-assemble into chimeric virus-like particles (VLPs), which were similar in size and morphology to native PCV2 particles. In addition, an effective affinity purification system for the purification of chimeric VLPs, referred to Gram-positive Enhancer Matrix-protein anchor domain-variable domain of heavy chain of heavy-chain antibody 8 (GEM-PAVHH8), was screened and identified. The PA-VHH8 fusion protein can specifically bind to GEM particles by covalent bond adsorption, which contributes to display Cap-S1D chimeric VLPs on the surface of GEM particles. Cap-S1D chimeric VLPs were successfully purified with the developed purification system and the purification efficiency was close to $90 \%$. What's more, the purified chimeric VLPs homemade vaccine induced a stronger humoral and cellular immune responses compared to the unpurified recombinant protein in pigs. Thus, the GEM-PA-VHH system provided an effective and safe technology for the preparation and purification of chimeric VLPs to prevent PEDV and PCV2 infection.
\end{abstract}

\section{Hosted file}

2020-5.5-TAED-Preparation and Purification of Porcine Circ.doc available at https: //authorea.com/users/319270/articles/449012-preparation-and-purification-of-porcinecircovirus-type-2-chimeric-virus-like-particles-by-a-new-affinity-purification-system 
A

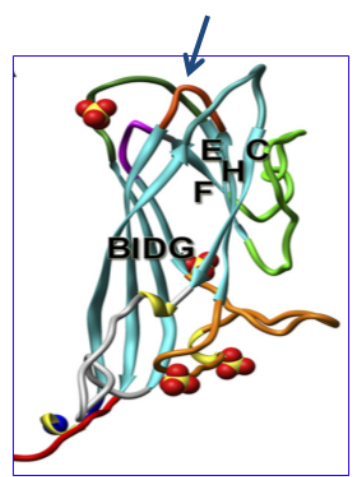

PCV2 Cap protein structure

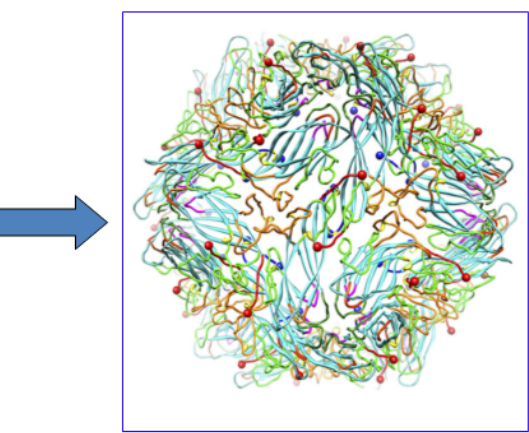

PCV2 virus-like particle structure

B

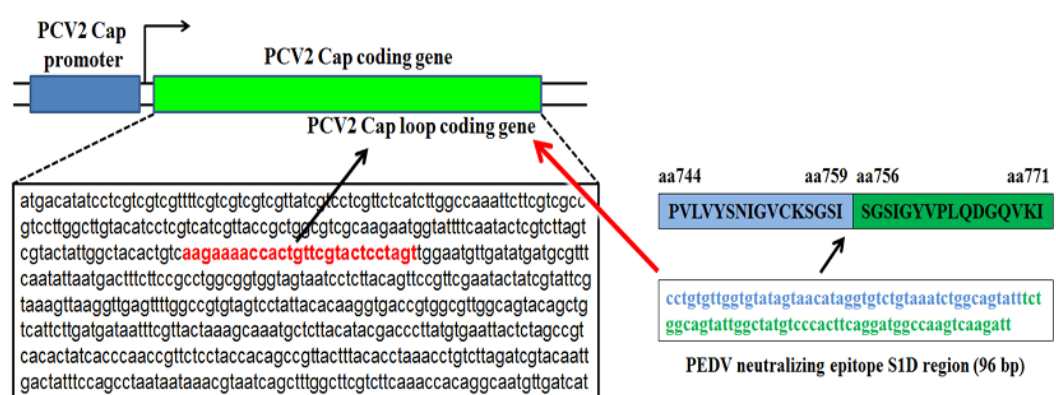

gactatttccagcctaataataaacgtaatcagctttggcttcgtcttca

gtaggtcttggtactgcattgaaaatagtattlacgaccaggattacaatattcgtattacaatgtacgtgc

aattccgtgaatttaatcttaaagatcctccattgaatccgtaa

$\mathcal{C}$

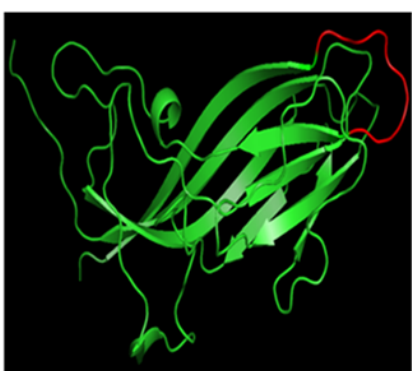

PCV2 Cap protein structure

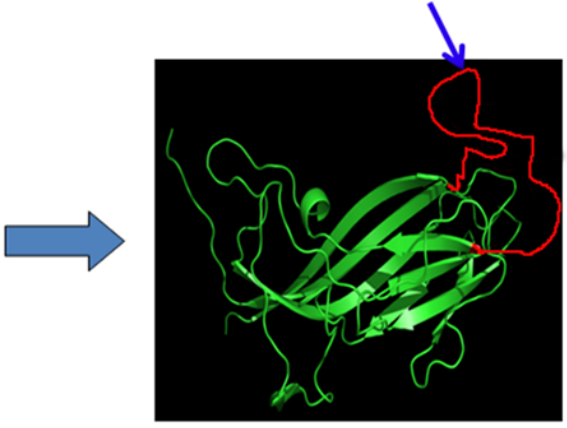

PCV2 Cap-S1D chimeric protein structure 
A

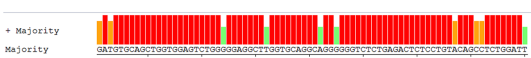

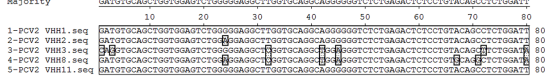

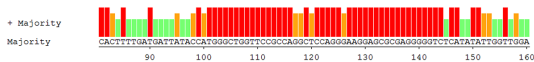

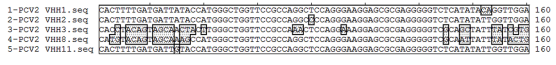

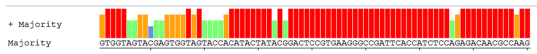

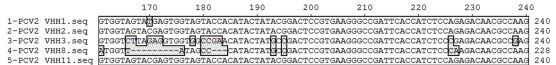

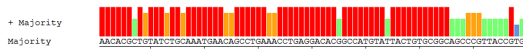

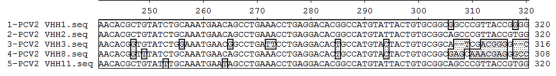

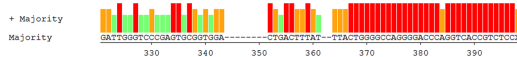

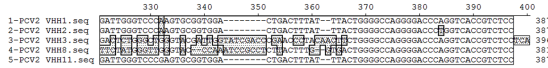

B

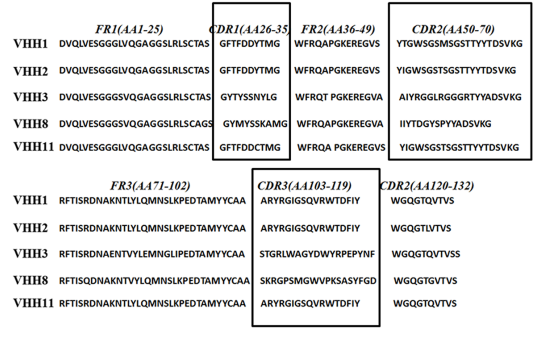

C

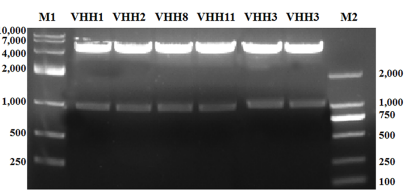

D

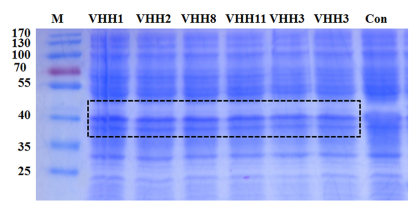

$\mathbf{E}$

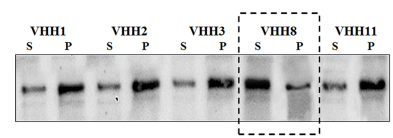

F

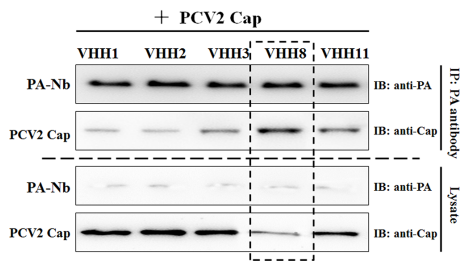


A
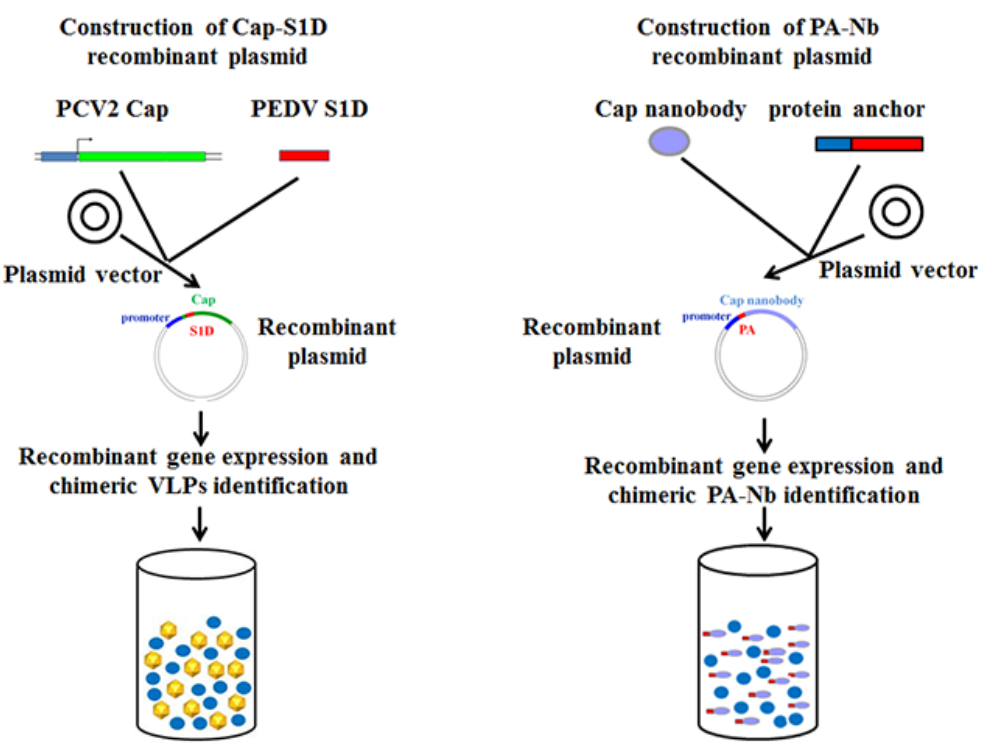

B

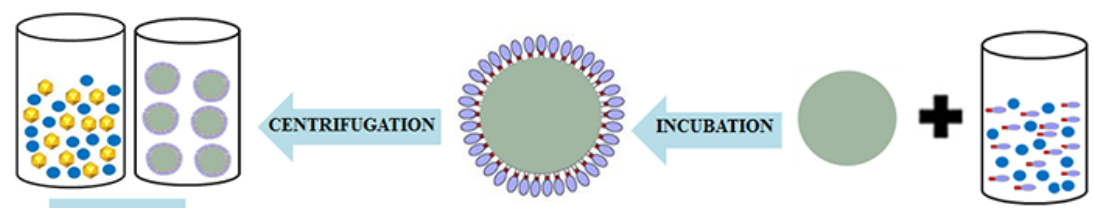

INCUBATION
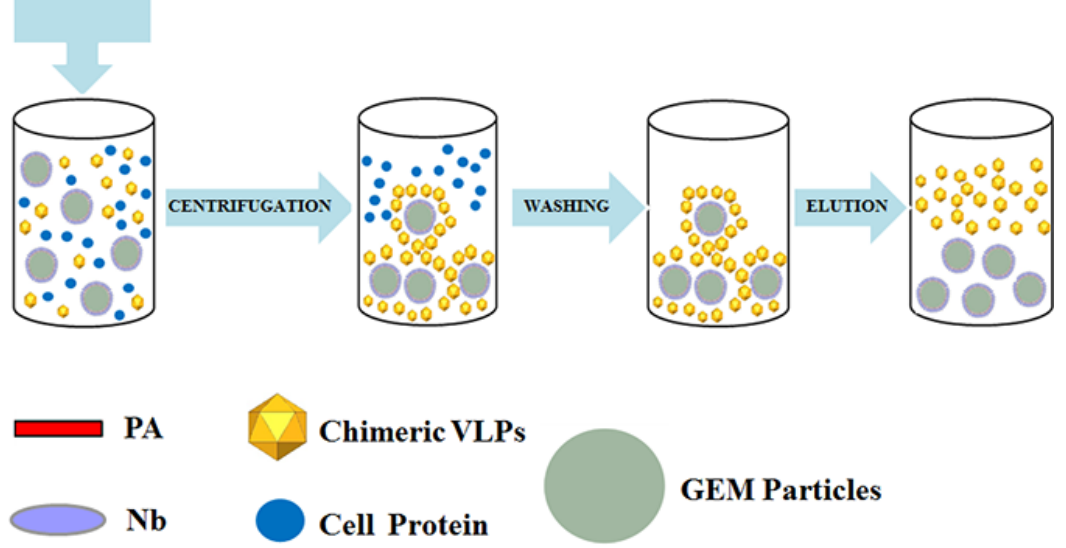


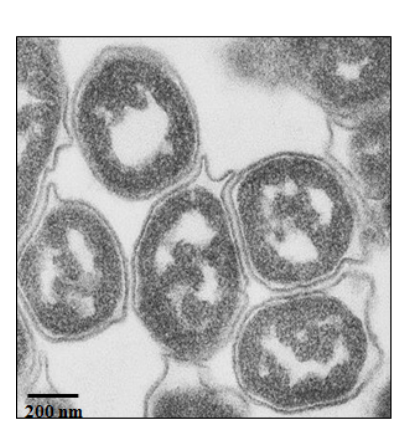

A

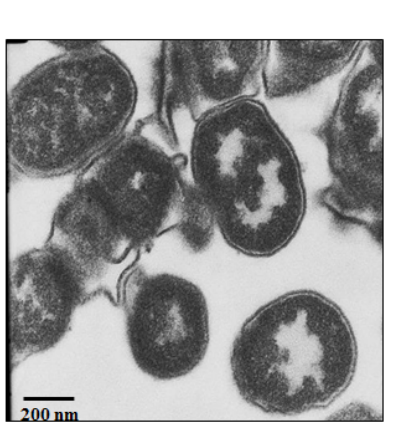

B

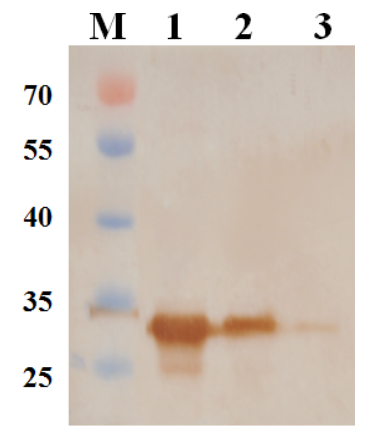

C

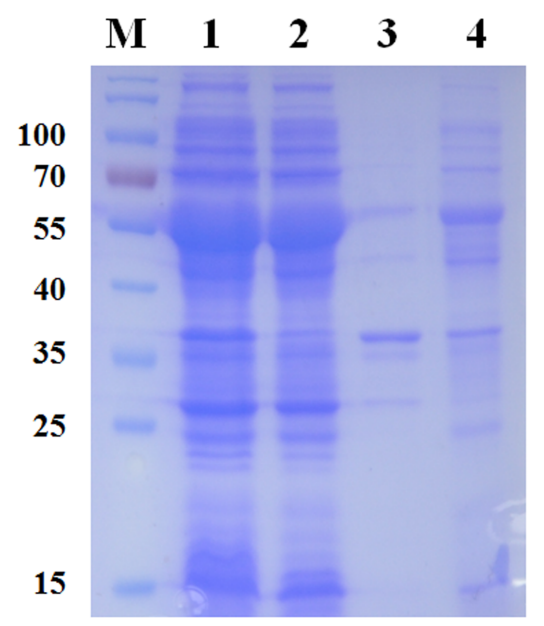

A
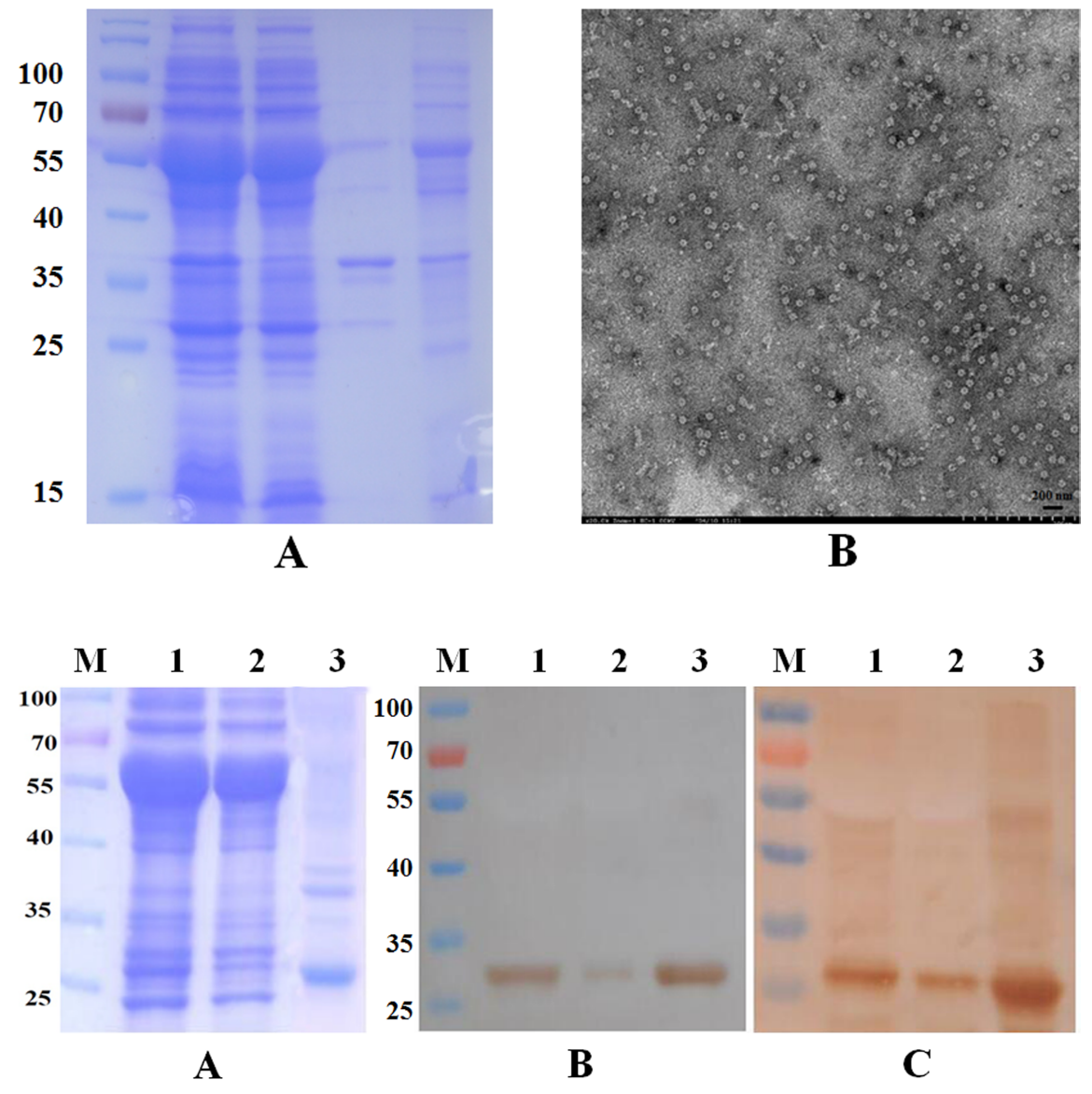


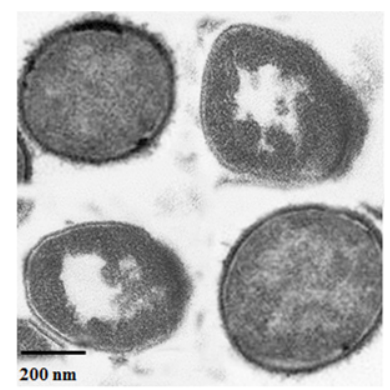

A

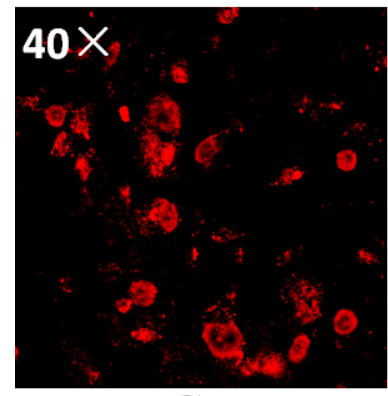

C

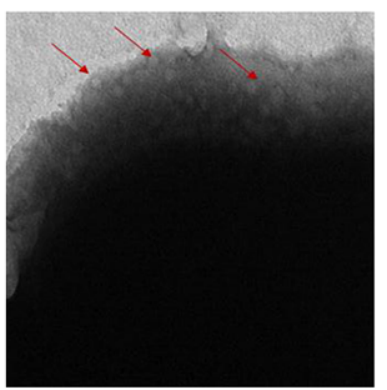

B

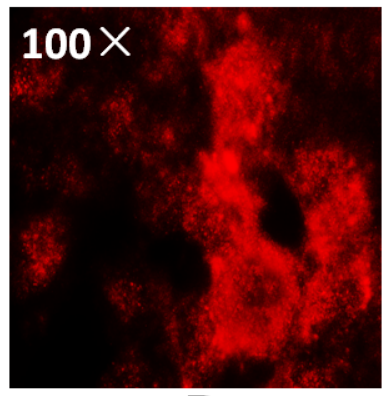

D
$40 \times$

E 


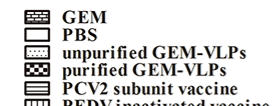

易 PEDV inactivated vaccine
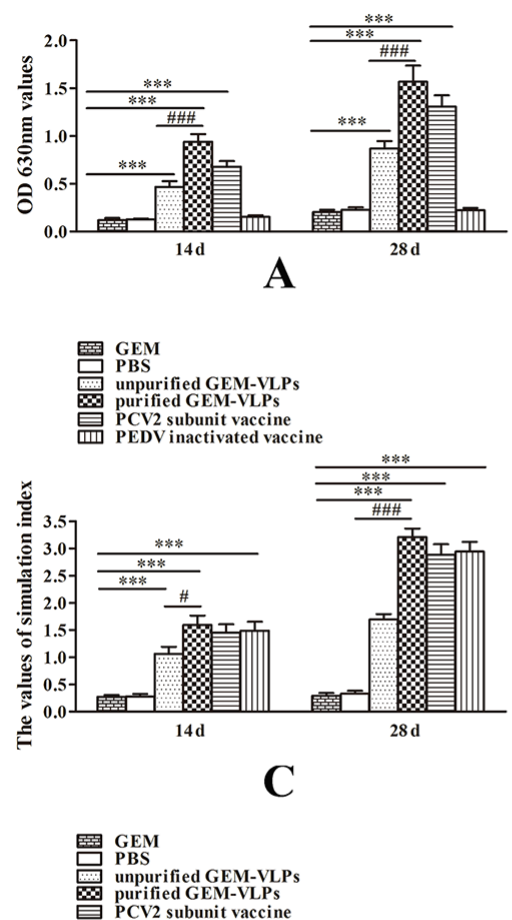

PCV2 subunit vaccine

昷 PEDV inactivated vaccine

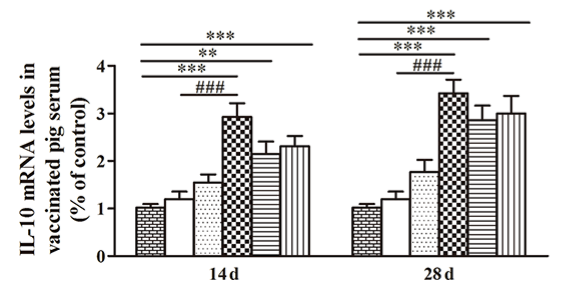

$\mathbf{E}$
置 GEM

署 unpurified GEM-VLPs

purified GEM-VLPs

昷 PEDV inactivated vaccine

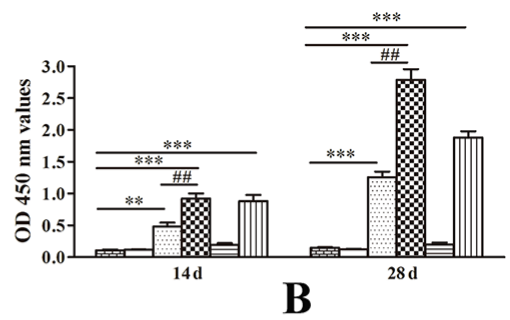

置 GEM

骷 unpurified GEM-VLPs

purified GEM-VLPs

PEDV inactivated vaccine

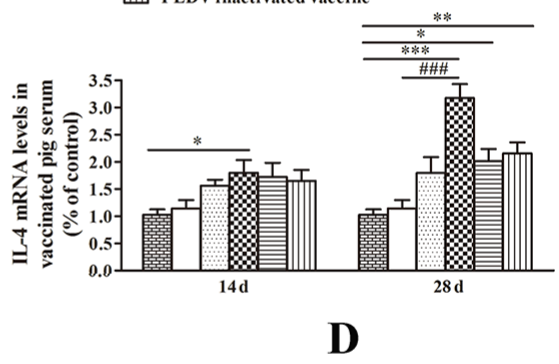

監 GEM

髙 unpurified GEM-VLPs

PCV2 subunit vaccine

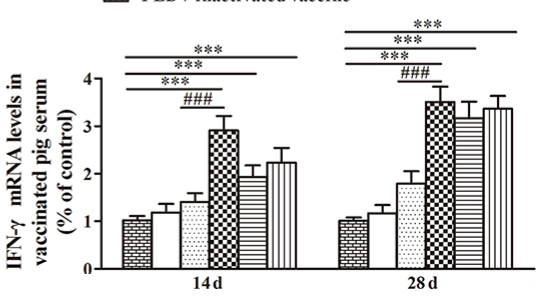

F

\section{Hosted file}

Table1.doc available at https://authorea.com/users/319270/articles/449012-preparationand-purification-of-porcine-circovirus-type-2-chimeric-virus-like-particles-by-a-newaffinity-purification-system

\section{Hosted file}

Table2.doc available at https://authorea.com/users/319270/articles/449012-preparationand-purification-of-porcine-circovirus-type-2-chimeric-virus-like-particles-by-a-newaffinity-purification-system 